Research Paper

\title{
UBE2O promotes hepatocellular carcinoma cell proliferation and invasion by regulating the AMPKa2/ mTOR pathway
}

Zhan Shi1,2\#, Runkun Liü\#, Qiliang Lu4, Zhi Zeng4, Yang Liu ${ }^{4}$, Junjun Zhao5, Xin Liu², Lijie Li6, Hui Huang7, Yingmin $\mathrm{Yao}^{3}$, Dongsheng Huang ${ }^{2 \varpi}$ and Qiuran $\mathrm{Xu}^{2 \llbracket}$

1. The Second Clinical Medical College, Zhejiang Chinese Medical University, Hangzhou 310053, China.

2. The Key Laboratory of Tumor Molecular Diagnosis and Individualized Medicine of Zhejiang Province, Zhejiang Provincial People's Hospital, Affiliated People's Hospital, Hangzhou Medical College, Hangzhou 310014, China.

3. Department of Hepatobiliary Surgery, The First Affiliated Hospital of Xi'an Jiaotong University, Xi'an 710061, China.

4. The Medical College of Qingdao University, Qingdao, 266071, China.

5. Graduate Department, Bengbu Medical College, Bengbu 233030, China.

6. Department of Obstetrics and Gynaecology, Affiliated Zhejiang Hospital, Zhejiang University School of Medicine, Hangzhou 310013, China.

7. Affiliated Quzhou People's Hospital, Zhejiang Chinese Medical University, Quzhou 324002, China.

\#These authors contributed equally to this work.

$\triangle$ Corresponding authors: Dr. Qiuran Xu, Tel/Fax: +86051785335800, E-mail: liuxin@hmc.edu.cn; Dr. Dongsheng Huang, Tel/Fax: +86051785335800, E-mail: dshuang@hmc.edu.cn.

() The author(s). This is an open access article distributed under the terms of the Creative Commons Attribution License (https://creativecommons.org/licenses/by/4.0/). See http://ivyspring.com/terms for full terms and conditions.

Received: 2021.05.27; Accepted: 2021.09.27; Published: 2021.10.11

\begin{abstract}
The ubiquitin-conjugating enzyme (E2) is a critical component of the ubiquitin-proteasome system and regulates hepatocarcinogenesis by controlling protein degradation. Ubiquitin-conjugating enzyme E2 O (UBE2O), a member of the E2 family, functions as an oncogene in human cancers. Nevertheless, the role of UBE2O in hepatocellular carcinoma (HCC) remains unknown yet. Here, we demonstrated that the UBE2O level was markedly upregulated in HCC compared with adjacent noncancerous tissues. UBE2O overexpression was also confirmed in HCC cell lines. UBE2O overexpression was prominently associated with advanced tumor stage, high tumor grade, venous infiltration, and reduced HCC patients' survivals. UBE2O knockdown inhibited the migration, invasion, and proliferation of HCCLM3 cells. UBE2O overexpression enhanced the proliferation and mobility of Huh7 cells. Mechanistically, UBE2O mediated the ubiquitination and degradation of AMP-activated protein kinase $\alpha 2$ (AMPK $\alpha 2)$ in HCC cells. UBE2O silencing prominently increased AMPKa2 level and reduced phosphorylated mechanistic target of rapamycin kinase (p-mTOR), MYC, Cyclin DI, HIFla, and SREBPI levels in HCCLM3 cells. UBE2O depletion markedly activated the AMPK $\alpha 2 / m$ TOR pathway in Huh7 cells. Moreover, AMPKa2 silencing reversed UBE2O downregulation-induced mTOR pathway inactivation. Rapamycin, an inhibitor of mTOR, remarkably abolished UBE2O-induced mTOR phosphorylation and HCC cell proliferation and mobility. To conclude, UBE2O was highly expressed in HCC and its overexpression conferred to the poor clinical outcomes of patients. UBE2O contributed to the malignant behaviors of HCC cells, including cell proliferation, migration, and invasion, by reducing $A M P K \alpha 2$ stability and activating the mTOR pathway.
\end{abstract}

Key words: Hepatocellular carcinoma; UBE2O; AMPKa2; mTOR pathway; Tumor progression

\section{Introduction}

The most recent IARC data shows that liver cancer is the fifth commonest cancer and the $2^{\text {nd }}$ leading cause of cancer-related deaths in China [1]. About $90 \%$ of liver cancer is hepatocellular carcinoma (HCC), which has a poor prognosis with a five-year survival of less than $20 \%$ after surgery in China [2]. To improve the clinical outcomes of patients with HCC, researchers focus on the investigation of HCC pathogenesis and the discovery of new targeted drugs for HCC. 
Ubiquitination is an important post-translational modification of protein and plays an essential role in controlling substrate degradation [3]. The ubiquitin proteasome system (UPS) contains ubiquitinactivating enzyme (E1), ubiquitin-conjugating enzyme (E2), ubiquitin ligase (E3), proteasome and deubiquitinating enzymes (DUBs) [4]. E1 activates ubiquitin (Ub) using ATP and transfers it to E2, E2 interacts with a specific E3 and transfers $\mathrm{Ub}$ to the target protein, and finally the ubiquitinated substrate is degraded by $26 \mathrm{~S}$ proteasome [5]. Moreover, DUBs remove $\mathrm{Ub}$ from substrate proteins to reverse the function of $\mathrm{Ub}$ ligases [6]. Increasing researches demonstrate the critical functions of ubiquitin modifying enzymes in the occurrence and progression of human HCC [7-9]. For instance, the E3-ubiquitin ligase tumor necrosis factor receptorassociated factor 7 (TRAF7) is highly expressed in HCC, and its overexpression predicts the poor prognosis of HCC [10]. TRAF7 directly targets Krüppel-like factor 4 (KLF4) for ubiquitin proteolysis to facilitate HCC progression [10]. F-box and WD repeat domain containing 7 (FBXW7) is identified as a tumor-suppressive factor and exerts an inhibitory role in HCC cell growth by regulating Yes-associated protein (YAP) degradation [8]. The upregulation of ubiquitin-conjugating enzyme E2 S (UBE2S) and its association with poor prognosis are reported by a previous study [11]. UBE2S contributes to HCC progression by interacting with tripartite motifcontaining 28 (TRIM28) and subsequently promoting the ubiquitination of p27 [12]. UBE2L3 functions as an oncogene and contributes to the proliferation and apoptosis resistance of tumor cells by suppressing the glycogen synthase kinase 3 beta (GSK3 $\beta$ )/p65 pathway in HCC [13].

Recent studies report the oncogenic or tumorsuppressive role of UBE2O in human cancers [14-19]. For example, UBE2O mediates the multi-monoubiquitination of BRCA1-associated protein 1 (BAP1) and subsequently reduces its cytoplasmic retention [15]. UBE2O participates in mixed-lineage leukemia (MLL) progression by regulating MML protein degradation [14]. UBE2O targets c-Maf for ubiquitination and degradation to repress the tumor growth of myeloma [17]. Mxi1 is identified as a substrate of UBE2O and mediates the tumorpromoting role of UBE2O in lung cancer [19]. AMPactivated protein kinase a2 (AMPKa2) is a classical target protein of $\mathrm{UBE} 2 \mathrm{O}$ in skeletal muscle and human cancers $[16,18,20]$. UBE2O activates the mechanistic target of rapamycin kinase (mTOR) signaling pathway by ubiquitinating and destabilizing AMPKa2 in human cancers [16, 18]. Nevertheless, the role of UBE2O in HCC and the underlying mechanism are unclear yet.

Here, we determined UBE2O expression in HCC and adjacent nontumor tissues and analyzed its prognostic significance. We investigated the effects of UBE2O on cell migration, invasion, and proliferation. The AMPKa2/mTOR pathway regulated by UBE2O was confirmed in HCC cells. Our data showed that the level of UBE2O was reduced in HCC specimens and associated with a poor prognosis. UBE2O enhanced the malignant behaviors of tumor cells by regulating the AMPKa2/mTOR pathway in HCC.

\section{Material and methods}

\section{HCC patients and specimens}

We collected eighty pairs of tumors and adjacent nontumor tissues from HCC patients who underwent surgical resection at the $1^{\text {st }}$ Affiliated Hospital of Xi' an Jiaotong University after obtaining written informed consent. All patients were not treated with pre-operative treatment and had complete clinical information, including follow-up data. The pathologists confirmed all specimens, and we subsequently maintained them in $-80^{\circ} \mathrm{C}$ for RT-qPCR analysis and fixed them with $10 \%$ formalin for immunohistochemistry. The Ethics Committee of the $1^{\text {st }}$ Affiliated Hospital of Xi'an Jiaotong University approved the current study protocols. Table 1 contains HCC patients' clinical characteristics.

\section{Cell culture and plasmid transfection}

We previously preserved five human HCC cell lines (HCCLM3, Hep3B, HepG2, MHCC97H, and Huh7) in our lab [21]. The normal hepatic cell line MIHA was obtained from bnbio (Beijing, China). All cell lines were cultured in DMEM medium (Gibco, Gaithersburg, MD, USA) supplemented with 10\% fetal bovine serum (FBS, Gibco), streptomycin (100 $\mathrm{mg} / \mathrm{mL})$, and penicillin $(100 \mathrm{U} / \mathrm{mL})$ in a $5 \% \mathrm{CO}_{2}$ incubator at $37^{\circ} \mathrm{C}$. We generated UBE2O expression plasmid by inserting cDNA into the pcDNA3.1 vector (Invitrogen, Carlsbad, CA, USA). UBE2O shRNAs (sh-UBE2O\#1 and sh-UBE2O\#2), AMPKa2 shRNA (sh-AMPKa2), and nontargeting shRNA (sh-NC) were provided by GenePharma (Shanghai, China). The vectors were transfected into HCC cells using Qiagen Effectene transfection reagent (Valencia, CA, USA). The shRNA sequences were shown in Supplementary Table 1.

\section{RT-qPCR analysis}

Total RNA was extracted using Trizol reagent (Invitrogen), and the RNA was reverse-transcribed into cDNA according to the manufacturer's instructions of PrimeScript RT Kit (Takara, Japan). RT-qPCR was performed using a fast SYBR Green 
PCR kit (Applied Biosystems, Foster City, CA, USA) and ABI PRISM 7300 RT-PCR system (Applied Biosystems) on the Synthesis of cDNA, and the reaction was run in triplicate. GAPDH was used as an internal reference. The relative gene expression was calculated by the $2^{-\Delta \Delta \mathrm{Ct}}$ method. The primer sequences were shown in Supplementary Table 1.

Table 1. Clinicopathological correlation of UBE2O expression in human hepatocellular carcinoma

\begin{tabular}{|c|c|c|c|c|}
\hline \multirow[t]{2}{*}{ Characteristics } & \multirow[t]{2}{*}{$\mathrm{n}=80$} & \multicolumn{2}{|l|}{ UBE2O } & \multirow[t]{2}{*}{$P$} \\
\hline & & $\begin{array}{l}\text { Low expression } \\
(\mathrm{n}=39)\end{array}$ & $\begin{array}{l}\text { High expression } \\
(\mathrm{n}=41)\end{array}$ & \\
\hline \multicolumn{2}{|l|}{ Age (years) } & & & 0.352 \\
\hline$<50$ & 35 & 15 & 20 & \\
\hline$\geq 50$ & 45 & 24 & 21 & \\
\hline \multicolumn{2}{|l|}{ Sex } & & & 0.481 \\
\hline Male & 63 & 32 & 31 & \\
\hline Female & 17 & 7 & 10 & \\
\hline \multicolumn{2}{|l|}{ HBV infection } & & & 0.527 \\
\hline No & 28 & 15 & 13 & \\
\hline Yes & 52 & 24 & 28 & \\
\hline \multicolumn{3}{|c|}{ Serum AFP level (ng/mL) } & & 0.385 \\
\hline$<20$ & 27 & 15 & 12 & \\
\hline$\geq 20$ & 53 & 24 & 29 & \\
\hline \multicolumn{2}{|l|}{ Tumor size (cm) } & & & 0.267 \\
\hline$<5$ & 26 & 15 & 11 & \\
\hline$\geq 5$ & 54 & 24 & 30 & \\
\hline \multicolumn{3}{|c|}{ No. of tumor nodules } & & 0.185 \\
\hline 1 & 65 & 34 & 31 & \\
\hline$\geq 2$ & 15 & 5 & 10 & \\
\hline \multicolumn{2}{|l|}{ Cirrhosis } & & & 0.185 \\
\hline No & 35 & 20 & 15 & \\
\hline Yes & 45 & 19 & 26 & \\
\hline \multicolumn{2}{|c|}{ Venous infiltration } & & & $0.041^{*}$ \\
\hline No & 44 & 26 & 18 & \\
\hline Yes & 36 & 13 & 23 & \\
\hline \multicolumn{3}{|c|}{ Edmondson-Steiner grade } & & $0.037^{*}$ \\
\hline I+II & 57 & 32 & 25 & \\
\hline III+IV & 23 & 7 & 16 & \\
\hline \multicolumn{2}{|l|}{ TNM stage } & & & $0.004^{*}$ \\
\hline I+II & 63 & 36 & 27 & \\
\hline III+IV & 17 & 3 & 14 & \\
\hline
\end{tabular}

$\mathrm{HBV}$, hepatitis B virus; $\mathrm{AFP}$, alpha-fetoprotein; TNM, tumor-node-metastasis. The "low" or "high" expression of UBE2O level was defined according to the cut-off value, which was defined as the median value of the cohort of patients tested.

"Statistically significant.

\section{Immunohistochemical (IHC) staining}

According to previously described protocols, we used forty samples containing both HCC and adjacent nontumor tissues for the immunohistochemical staining with anti-UBE2O antibody (1:200, 15812-1-AP, Proteintech, Wuhan, China) [22]. Two pathologists examined the slides in a double-blind fashion. We randomly selected five high-power fields of view for each section. The intensity of staining was scored as 0 (negative), 1 (weak), 2 (moderate), and 3 (strong), and we scored the percentage of UBE2O positive tumor cells as $1(0-25 \%), 2(26-50 \%)$, $3(51-75 \%)$, and $4(75-100 \%)$ [19]. The IHC score was equal to the intensity score multiplied by the positive rate score.

\section{Western blotting}

The cells were lysed using RIPA buffer (Boster Biological Technology Ltd., Wuhan, China). The protein concentration was determined based on the manufacturer's instructions of the BCA protein assay kit (Boster). Proteins were separated by 10\% SDSPAGE, and the separated proteins were electrotransferred onto a PVDF membrane (Millipore, Bedford, MA, USA). The membrane was probed with diluted primary antibodies followed by overnight incubation at $4{ }^{\circ} \mathrm{C}$. The next day, the membrane was labeled with HRP-bound secondary antibody incubation (1:1000, Beyotime, Shanghai, China) and detected using an ECL system (Millipore). The resulting bands were scanned using the Amersham Imager 680 machine (GE Healthcare Life Sciences, Pittsburgh, PA, USA). The used primary antibodies were listed in Supplementary Table 2.

\section{Cell proliferation assay}

For the cell counting kit- 8 (CCK- 8 ) assay, $2 \times 10^{4}$ transfected cells were resuspended in $1 \mathrm{~mL}$ complete DMEM medium and seeded into a 96-well plate (100 $\mu \mathrm{L}$ for each well). $10 \mu \mathrm{L}$ of CCK-8 solution (Beyotime) was added to each well of the plate and incubated for $4 \mathrm{~h}$. The absorbance at $450 \mathrm{~nm}$ was detected using a Multiskan FC microplate reader (Thermo Fisher Scientific). The EdU assay was carried out using the Cell-Light ${ }^{\mathrm{TM}}$ EdU Apollo®488 In Vitro Imaging Kit (RIBOBIO, Guangzhou, China) following the manufacturer's protocol, as previously described [23].

\section{Transwell assay}

For migration assay, $3 \times 10^{4}$ cells were resuspended in serum-free DMEM medium and subsequently placed in the upper chamber, while we added DMEM medium containing 10\% FBS to the lower chamber. The cells were cultured at $37^{\circ} \mathrm{C}$ for 24 $\mathrm{h}$. The total migrated cells to the lower chamber were fixed with $4 \%$ paraformaldehyde, stained with $0.1 \%$ crystal violet, and observed under the microscope. For invasion assay, the transwell chamber was covered with Matrigel (BD Biosciences, San Jose, CA, USA). Other operations were the same as the migration assay. The number of migrated and invaded cells were counted to evaluate cell migration and invasion abilities.

\section{Ubiquitination detection}

HCC cells were lysed with an IP buffer containing $0.5 \%$ NP40. Cell lysates containing equal volumes and equal amounts of proteins were incubated with $4 \mu \mathrm{g}$ anti-AMPKa2 antibody (18167- 
1-AP, Proteintech) and $30 \mu \mathrm{l}$ slurry of Protein G Sepharose (GE Healthcare Life Sciences) for overnight to pull down AMPKa2 and its associated proteins. The beads were precipitated by centrifugation on the following day, and $\mathrm{Ub}$ in the precipitates was quantitated by WB using anti-Ub antibody (1:2000, PTM-1107, PTM BIO, Hangzhou, China).

\section{TCGA data analysis}

The expression of UBE2O and its prognostic significance in the HCC cohort from the TCGA database was performed using the gene expression profiling interactive analysis (GEPIA) webserver (http://gepia.cancer-pku.cn/) according to the manufacturer's protocol [24]. The correlation between UBE2O level and tumor stage and tumor grade of HCC was analyzed using UALCAN (http://ualcan. path.uab.edu/analysis.html) [25].

\section{Statistical analysis}

The analysis of variance (ANOVA) with Tukey's multiple comparison test, Student's $t$-test, chi-squared test, and Mann-Whitney U-test were carried out using GraphPad Prism version 8 (GraphPad Inc., San Diego, CA, USA). The survival of two HCC subgroups was compared by the Kaplan-Meier method and log-rank test. Results from at least three independent repeats were shown as mean \pm S.D. $\mathrm{P}<0.05$ was considered statistically significant.

\section{Results}

\section{The overexpression of UBE2O is correlated with HCC patients' survivals}

Initially, our RT-qPCR data demonstrated that UBE2O expression was prominently increased in HCC tissue samples compared with adjacent noncancerous tissues $(\mathrm{P}<0.0001$, Figure $1 \mathrm{~A})$. Consistent with our data, TCGA data from the GEPIA web server [24] also indicated that UBE2O expression in HCC was markedly higher than that in normal tissues $(\mathrm{P}<0.05$, Supplementary Figure $1 \mathrm{~A})$. We used forty samples containing both HCC and adjacent nontumor tissues for the immunohistochemical staining of UBE2O. The IHC score of UBE2O in HCC was remarkably higher than in adjacent nontumor tissues $(\mathrm{P}<0.0001$, Figure $1 \mathrm{~B})$. The upregulated expression of UBE2O was verified in HCC cell lines, including HCCLM3, Huh7, Hep3B, HepG2, and MHCC97H compared to MIHA cells $(\mathrm{P}<0.05$, Figure 1C). Table 1 showed that the high level of UBE2O was associated with advanced TNM stage $(\mathrm{P}=0.004)$, venous infiltration $(\mathrm{P}=0.041)$, and high Edmondson-Steiner grade $(\mathrm{P}=0.037)$. The higher expression of UBE2O was detected in advanced and poorly differentiated HCCs, as suggested by TCGA

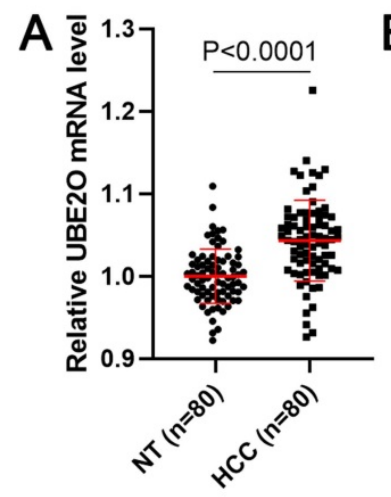

C

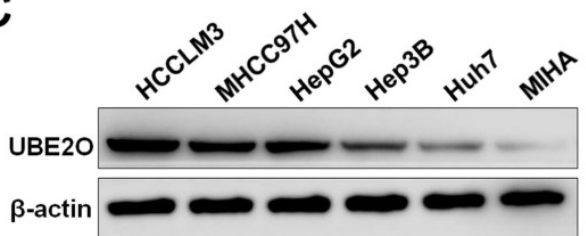

B
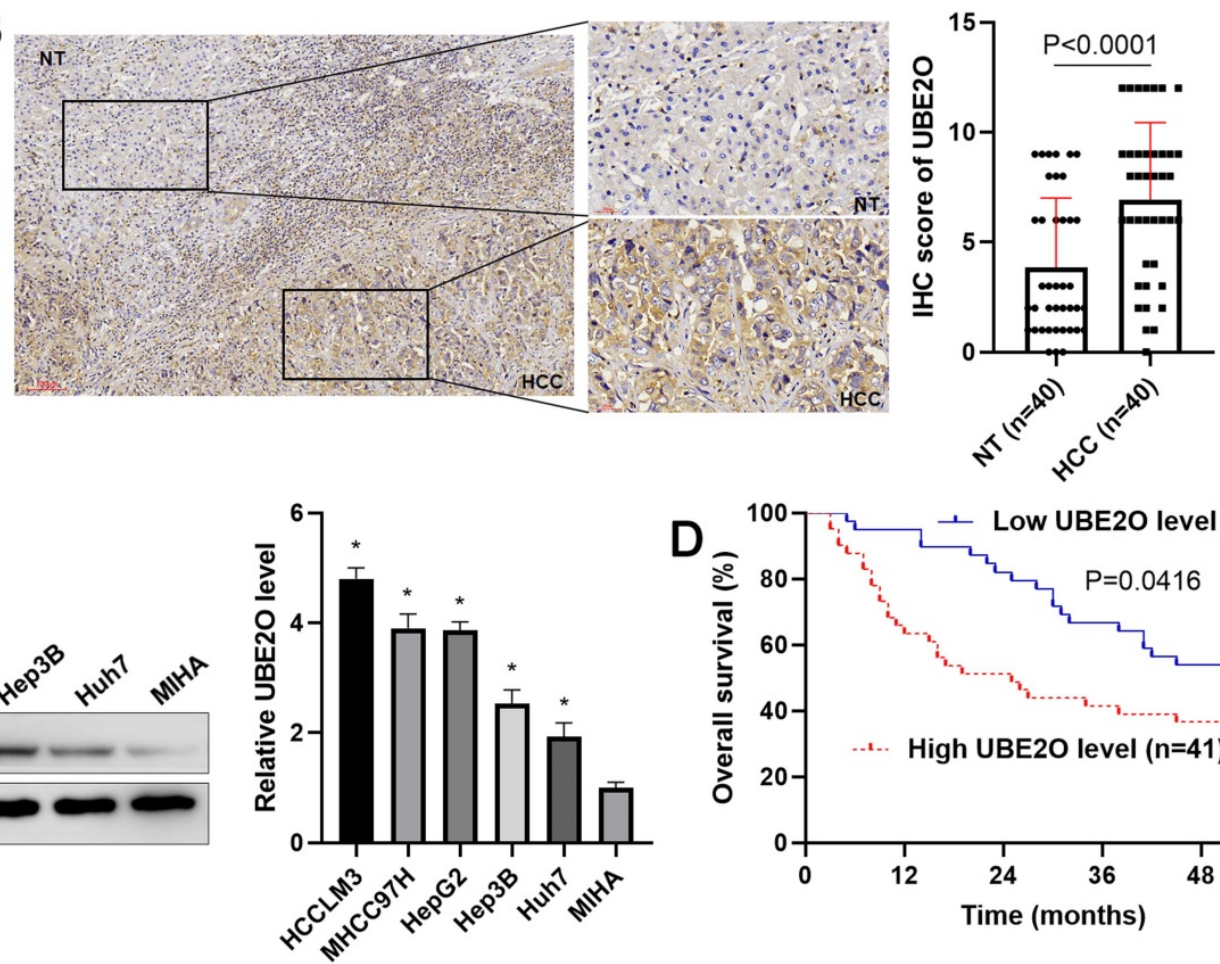

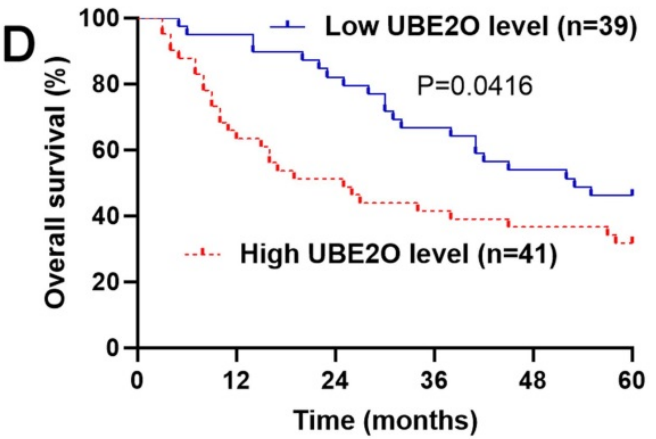

Figure 1. The expression and survival analysis of UBE2O in HCC. (A) The levels of UBE2O mRNA were detected in 80 pairs of HCC and nontumor (NT) tissues. (B) Left: The presentative result of UBE2O staining in a sample containing both $\mathrm{HCC}$ and adjacent NT tissues. Right: The IHC score of UBE2O in HCC ( $\mathrm{n}=40$ ) was remarkably higher than that in adjacent NT tissues $(n=40)$. (C) UBE2O expression was assessed in MIHA, HCCLM3, Huh7, HepG2, Hep3B, and MHCC97H cells. $n=$ three independent repeats, $* P<0.05$. (D) We compared the survival of HCC patients with low $(n=39)$ or highly $(n=41)$ expressed UBE2O. 
data from the UALCAN website [25] $(\mathrm{P}<0.05$, Supplementary Figure 1B). Next, our follow-up results and TCGA data consistently suggested that the increased level of UBE2O indicated the poor prognosis of HCC $(\mathrm{P}<0.05$, Figure $1 \mathrm{D}$ and Supplementary Figure 1C). Accordingly, our data showed UBE2O as a potential predictive marker for HCC prognosis.

\section{UBE2O facilitates the proliferation and invasion of HCC cells}

Two independent shRNA constructs downregulated UBE2O expression in HCCLM3 cells $(\mathrm{P}<0.05$, Figure $2 \mathrm{~A})$. The viability of HCC cells was significantly repressed by UBE2O knockdown $(\mathrm{P}<0.05$, Figure $2 \mathrm{~B})$, as suggested by the CCK- 8 assay. $\mathrm{UBE} 2 \mathrm{O}$ silencing markedly reduced the percentage of
EdU positive HCC cells $(\mathrm{P}<0.05$, Figure $2 \mathrm{C})$ and repressed cell clone formation ability $(\mathrm{P}<0.05$, Supplementary Figure 2). Transwell assays showed that UBE2O depletion remarkably reduced the number of migrated and invaded HCC cells $(\mathrm{P}<0.05$, Figure 2D). Next, UBE2O was ectopically expressed in Huh7 cells $(\mathrm{P}<0.05$, Figure $3 \mathrm{~A})$. The functional assays revealed that UBE2O overexpression facilitated the migration, invasion, and proliferation of HCC cells $(\mathrm{P}<0.05$, Figure 3B-3D and Supplementary Figure 2). The effects of UBE2O alteration also confirmed in MHCC97H and Hep3B cells, respectively $(\mathrm{P}<0.05$, Supplementary Figure 3). Therefore, these results suggested that UBE2O enhanced the malignant behaviors of HCC cells.
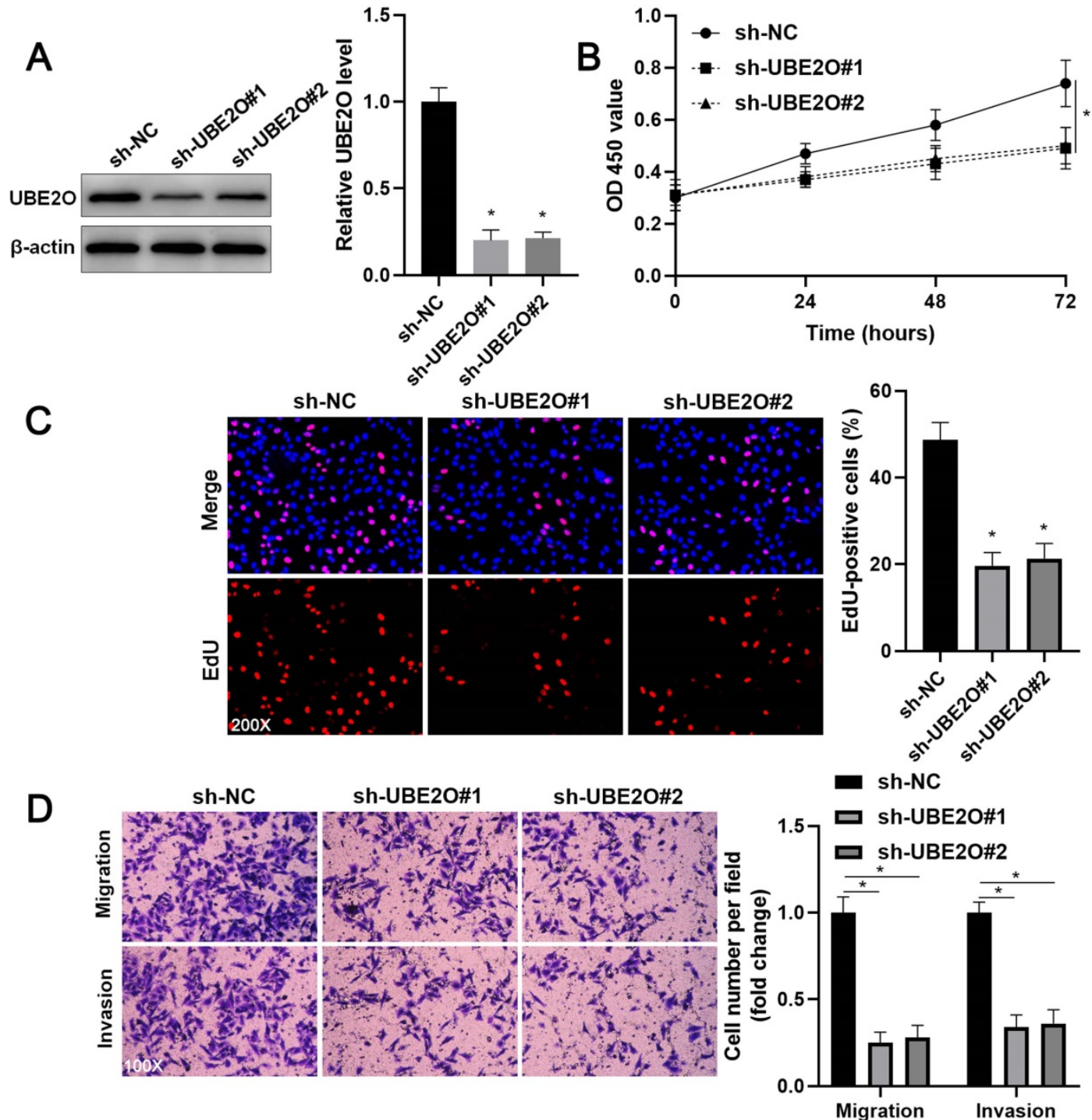

Figure 2. UBE2O knockdown inhibits HCCLM3 cell proliferation and mobility. (A) HCCLM3 cells were transfected with nontargeting shRNA (sh-NC) or UBE2O shRNAs (sh-UBE2O\#1 and sh-UBE2O\#2) and analyzed by immunoblotting for UBE2O expression at $72 \mathrm{~h}$ after transfection. (B) UBE2O silencing repressed HCCLM3 cell viability, as revealed by the CCK-8 assay. (C) EdU assay confirmed that UBE2O knockdown decreased HCCLM3 cell proliferation at $72 \mathrm{~h}$ after transfection. (D) The number of migrated and invaded HCCLM3 cells was reduced by UBE2O depletion at $72 \mathrm{~h}$ after transfection. $\mathrm{n}=$ three independent repeats, $* \mathrm{P}<0.05$. 

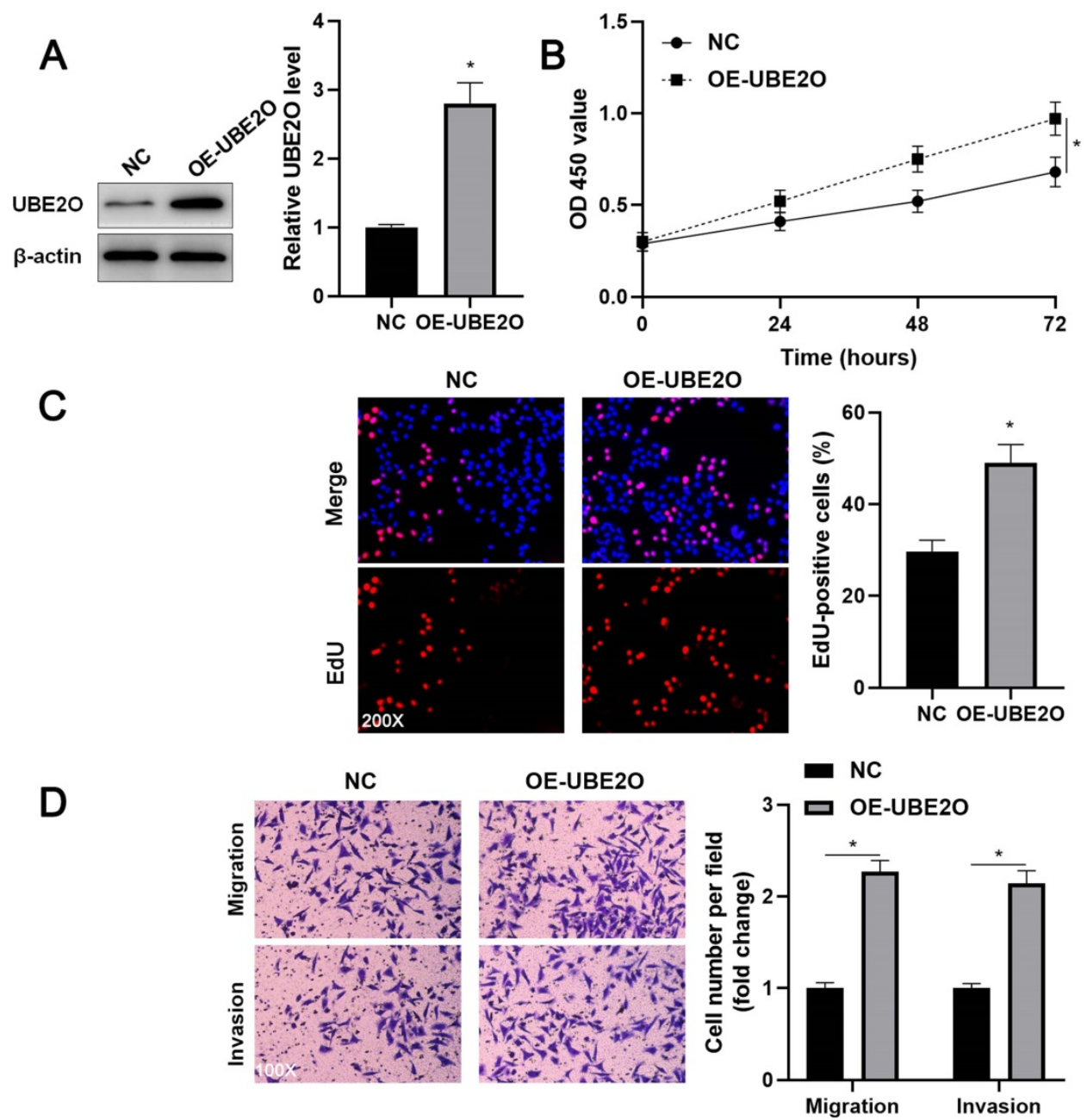

Figure 3. UBE2O overexpression promotes Huh7 cell proliferation and mobility. (A) Huh7 cells were transfected with pcDNA3.1 vector carrying UBE2O (OE-UBE2O) or empty vector (NC) and analyzed by immunoblotting for UBE2O expression at $72 \mathrm{~h}$ after transfection. (B) UBE2O overexpression facilitated Huh7 cell viability, as revealed by the CCK-8 assay. (C) EdU assay confirmed that UBE2O upregulation enhanced Huh7 cell proliferation at $72 \mathrm{~h}$ after transfection. (D) The number of migrated and invaded Huh7 cells was increased by UBE2O overexpression at $72 \mathrm{~h}$ after transfection. $\mathrm{n}=$ three independent repeats, $* \mathrm{P}<0.05$.

\section{UBE2O activates the $\mathrm{mTOR}$ pathway by reducing AMPKa2 level}

AMPKa2, a classical substrate of UBE2O, is downregulated in HCC and participates in tumor progression by regulating the mTOR pathway [26, 27]. Thus, we intended to confirm whether UBE2O controlled AMPKa2 abundance in HCC. As expected, we found that UBE2O knockdown significantly increased while UBE2O overexpression markedly reduced AMPKa2 protein level $(\mathrm{P}<0.05$, Figure $4 \mathrm{~A}$ and $4 \mathrm{C}$ ) without altering AMPKa2 mRNA level in HCC cells (Supplementary Figure 4A). UBE2O knockdown reduced the ubiquitination of AMPKa2 in HCCLM3 cells (Supplementary Figure 4B). UBE2O-induced AMPKa2 downregulation was reversed by a proteasome inhibitor MG132 in Huh7 cells (Supplementary Figure 4C). Moreover, UBE2O positively regulated $\mathrm{p}$-mTOR level but not total mTOR expression in HCC cells $(\mathrm{P}<0.05$, Figure $4 \mathrm{~A}$ and $4 \mathrm{C})$. The target genes of the mTOR pathway, including p-p70S6K, p-4EBP1, MYC, Cyclin D1, HIF1a, and SREBP1, were also positively regulated by UBE2O in HCC cells $(\mathrm{P}<0.05$, Figure $4 \mathrm{~B}$ and $4 \mathrm{D}$ and Supplementary Figure 5). AMPKa2 knockdown markedly reversed UBE2O silencing-induced the mTOR pathway inactivation in HCCLM3 cells $(\mathrm{P}<0.05$, Figure $4 \mathrm{E}$ and $4 \mathrm{~F})$. Collectively, UBE2O regulated the AMPKa2/mTOR pathway in HCC cells.

\section{The $m$ TOR inhibitor blocks the oncogenic role of UBE2O in HCC cells}

To investigate whether UEB2O exerts a tumorpromoting role by activating the mTOR pathway, rapamycin (RAPA), a specific $\mathrm{mTOR}$ inhibitor, was used to treat UBE2O overexpressing HCCLM3 cells. As shown in Figure 5A, UBE2O-induced p-mTOR upregulation was prominently reduced by RAPA treatment $(\mathrm{P}<0.05)$. RAPA treatment significantly repressed cell proliferation in UBE2O overexpressing HCCLM3 cells, as indicated by CCK- 8 and EdU assays $(\mathrm{P}<0.05$, Figure $5 \mathrm{~B}$ and $5 \mathrm{C})$. Furthermore, 
RAPA treatment remarkably abrogated the promoting role of UBE2O in HCCLM3 cell mobility $(\mathrm{P}<0.05$, Figure 5D). Thus, UBE2O activated the mTOR pathway to promote HCC progression.

\section{Discussion}

The dysregulation of UBE2O and its clinical significance has been previously reported in several types of human cancers. UBE2O is highly expressed in lung cancer tissues, and its overexpression predicts poor prognosis [19]. The overexpression of UBE2O is confirmed in breast cancer tissues, and UBE2O expression is higher in patients with a poor prognosis and an increased risk of metastasis [18]. According to TCGA and GEO databases, UBE2O upregulation that is frequently detected in breast, bladder, liver, lung, esophageal, and head and neck cancer predicts poor clinical outcomes in breast, lung, and gastric cancer [16]. In this study, we verified that UBE2O expression was prominently elevated in HCC. Then, we revealed that the increased level of UBE2O was closely correlated with advanced tumor stage, venous infiltration, high tumor grade, and poor prognosis of HCC. Thus, UBE2O might be a promising novel prognostic biomarker of HCC. However, the sample size of this study is not large enough, and there is no validation cohort. We will expand the sample size and further clarify the clinical significance of UBE2O in the validation cohort. Previous studies show that epigenetic and transcriptional regulations are implicated in the dysregulation of UBE2O in human cancers $[18,28]$. Thus, it will be necessary to disclose further the mechanism underlying the upregulation of UBE2O in HCC.
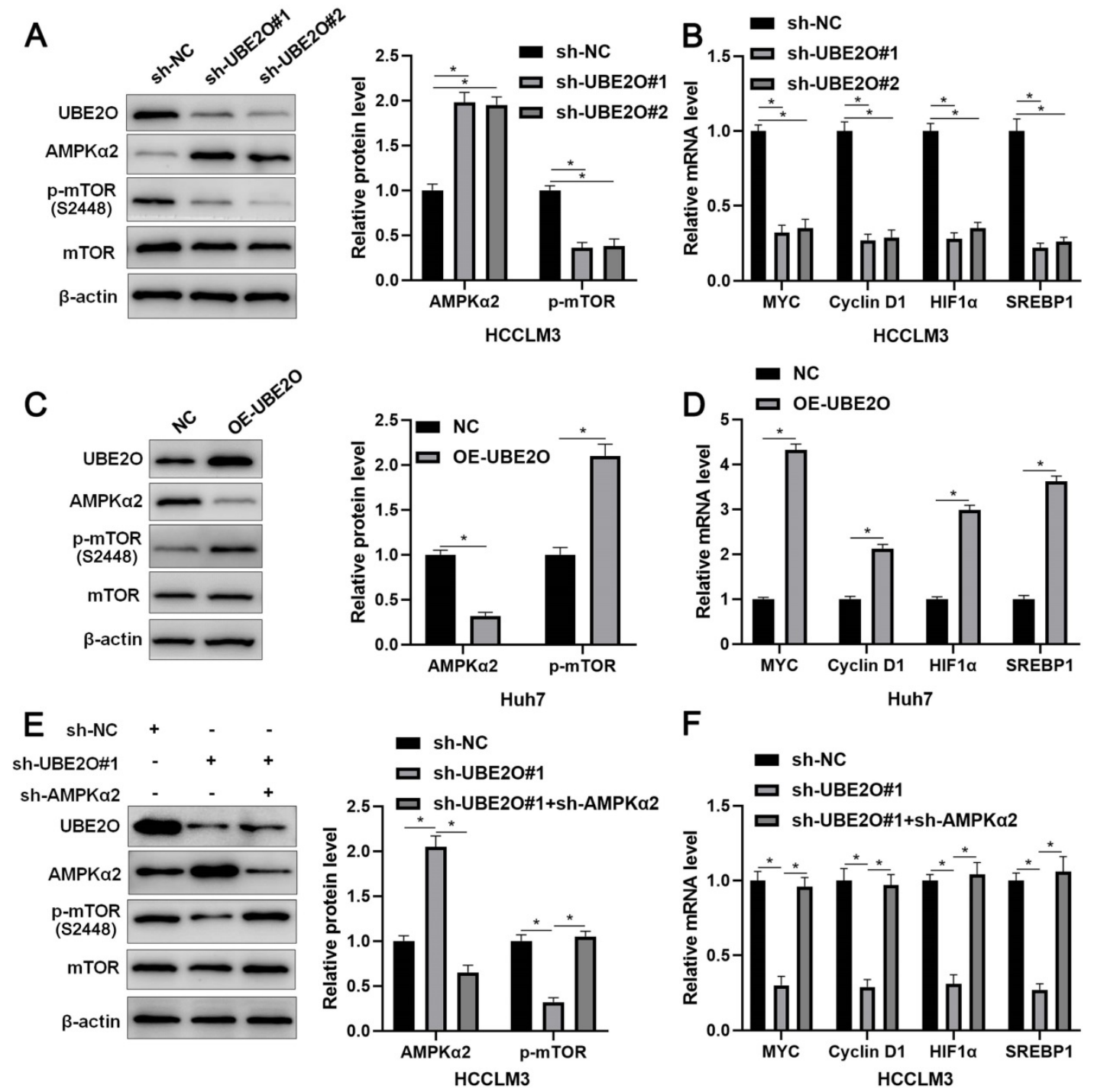

Figure 4. UBE2O regulates the AMPKa2/mTOR pathway in HCC. (A) HCCLM3 cells were transfected with sh-NC or UBE2O shRNAs (sh-UBE2O\#1 and sh-UBE2O\#2) and analyzed by immunoblotting for UBE2O, AMPKa2, p-mTOR, and mTOR levels at $72 \mathrm{~h}$ after transfection. (B) UBE2O knockdown reduced MYC, Cyclin D1, HIF1a, and SREBP1 mRNA levels in HCCLM3 cells at $72 \mathrm{~h}$ after transfection. (C) Huh7 cells were transfected with OE-UBE2O or NC and detected by immunoblotting for UBE2O, AMPKa2, p-mTOR, and mTOR levels at $72 \mathrm{~h}$ after transfection. (D) The ectopic expression of UBE2O upregulated MYC, Cyclin D1, HIF1 $\alpha$, and SREBP1 mRNA levels in Huh7 cells at $72 \mathrm{~h}$ after transfection. (E and F) Immunoblotting and RT-qPCR analysis found that the AMPKa2 knockdown reversed UBE2O silencing-induced the mTOR pathway inactivation in HCCLM3 cells at $72 \mathrm{~h}$ after transfection. $\mathrm{n}=$ three independent repeats, $* \mathrm{P}<0.05$. 

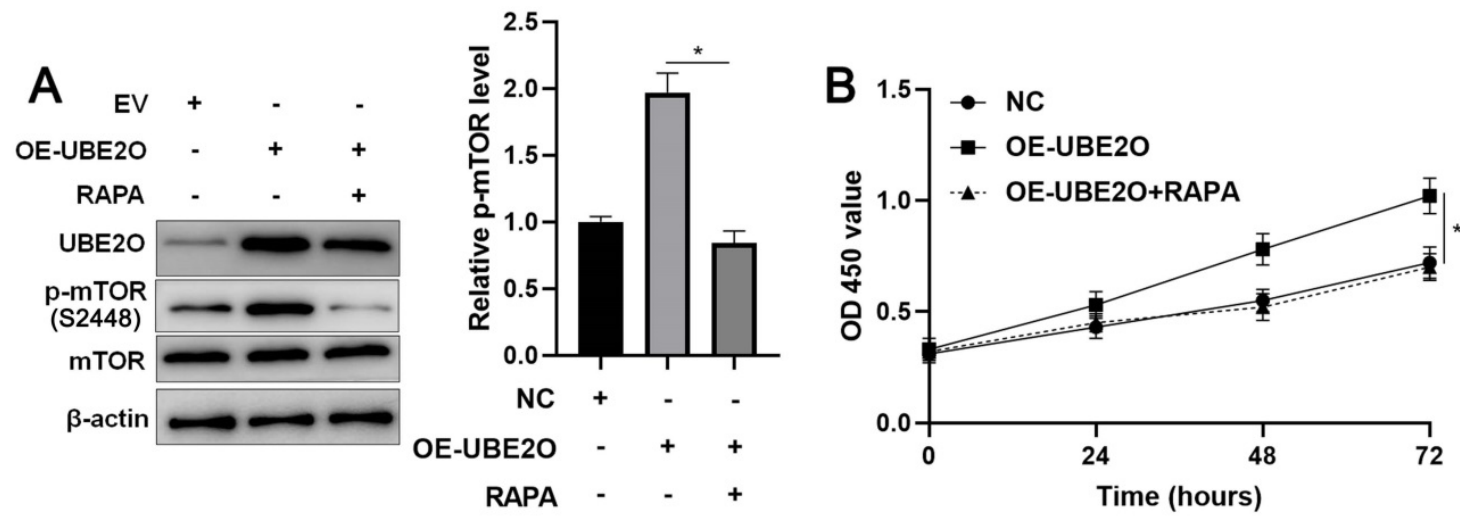

C
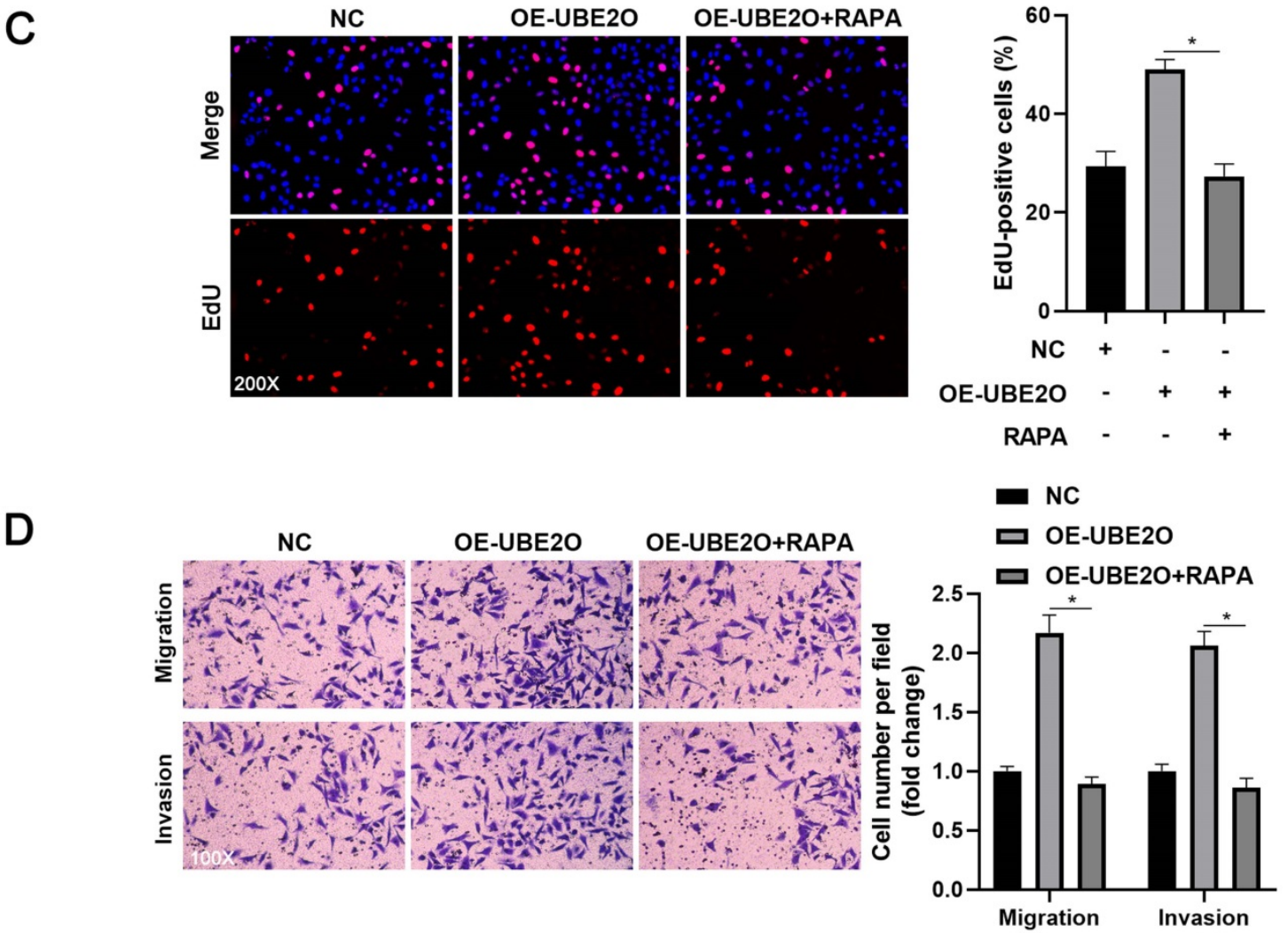

Figure 5. RAPA treatment reverses the promoting role of UBE2O in HCCLM3 cells. (A) UBE2O overexpressing HCCLM3 cells were treated with RAPA (10 $\mathrm{ng} / \mathrm{mL}$ ) for $48 \mathrm{~h}$, a specific mTOR inhibitor. UBE2O-induced p-mTOR upregulation was markedly reduced by RAPA treatment. (B) MTT, (C) EdU, and (D) transwell analyses demonstrated that RAPA treatment reversed the tumor-promoting role of UBE2O in HCCLM3 cells. $n=$ three independent repeats, $* P<0.05$.

Functional experiments confirmed that UBE2O acted as an oncogene in HCC and facilitated the proliferation and mobility of tumor cells. The UBE2O/AMPKa2 axis has been demonstrated in skeletal muscle and breast cancer [16, 18, 20]. UBE2O targets AMPKa2 for ubiquitination and degradation, thereby activating the mTOR pathway in breast cancer $[16,18]$. Here, we found that UBE2O also inversely regulated the AMPKa2 level in HCC cells without affecting the AMPKa2 mRNA level. We further confirmed the impact of UBE2O in the ubiquitination and degradation of AMPKa2 in HCC. Several other proteins, such as Mxi1, BAP1, c-Maf, and SMAD6, are recognized as substrates of UBE2O
$[15,17,19,29]$. Thus, it is worth checking the levels of these proteins after modulating UBE2O expression and discover novel targets for UBE2O in HCC cells. Next, we found that UBE2O positively modulated the mTOR pathway in HCC cells. Moreover, AMPKa2 knockdown markedly reversed UBE2O silencinginduced the inactivation of the mTOR pathway. $\mathrm{AMPKa} 2$ is previously reported to be low expressed in HCC and contributes to hepatocarcinogenesis by acetylating and stabilizing p53 [26]. AMPKa2 is also implicated in glycolic metabolism and chemoresistance of HCC [30]. Many studies have demonstrated that the mTOR pathway is one of the essential oncogenic pathways negatively regulated by 
AMPK in HCC [31-33]. mTOR forms a catalytic subunit of two distinct protein complexes, mTOR complex 1 (mTORC1) and mTORC2 [34]. Activation of mTORC1 most prominently results in phosphorylation of two downstream targets, p70S6K and 4EBP1, promoting protein synthesis [35]. 4EBP1 and p70S6K axes play critical and distinct roles in hepatocarcinogenesis [36]. The target genes of the mTOR pathway, such as MYC, Cyclin D1, HIF1a, and SREBP1, participate in many malignant behaviors of HCC cells [37-39]. Recent studies report that glutamine synthetase (GS), a transcriptional target of $\beta$-catenin, functions as a driver in HCC development by promoting p-mTOR (S2448) expression [40-42]. RAPA, a specific mTOR inhibitor, prominently abolished UBE2O-induced the mTOR pathway activation and HCC cell proliferation and mobility. Collectively, UBE2O exerted the pro-HCC effects by activating the AMPKa2/mTOR pathway. In this study, we did not provide animal experimental data to support the functional identification and molecular mechanism analysis of UBE2O in HCC. Therefore, it is necessary to perform the animal experiments in further study.

To conclude, we demonstrated the upregulation of UBE2O in HCC and verified their potential in predicting poor prognosis. UBE2O promoted the migration, invasion, and proliferation of tumor cells by activating the AMPKa2/mTOR pathway in HCC. UBE2O might be promising prognostic biomarkers and new therapeutic targets of HCC.

\section{Abbreviations}

HCC: hepatocellular carcinoma; TRAF7: tumor necrosis factor receptor-associated factor 7; KLF4: Krüppel-like factor 4; FBXW7: F-box and WD repeat domain containing 7; YAP: Yes-associated protein; UBE2S: ubiquitin-conjugating enzyme E2 S; TRIM28: tripartite motif-containing 28; GSK3ß: glycogen synthase kinase 3 beta; BAP1: BRCA1-associated protein 1; MLL: mixed-lineage leukemia; AMPKa2: AMP-activated protein kinase a2; mTOR: mechanistic target of rapamycin kinase.

\section{Supplementary Material}

Supplementary figures and tables.

http://www.medsci.org/v18p3749s1.pdf

\section{Acknowledgements}

This work was supported by grants from the National Natural Science Foundation of China (81874049), the Key Research and Development Program of Shaanxi (2020SF-068), the Zhejiang Provincial Natural Science Foundation of China (LY19H160036), and the Key Research and
Development Provincial Program (2020C03008).

\section{Competing Interests}

The authors have declared that no competing interest exists.

\section{References}

1. Sung H, Ferlay J, Siegel RL, Laversanne M, Soerjomataram I, Jemal A, et al. Global Cancer Statistics 2020: GLOBOCAN Estimates of Incidence and Mortality Worldwide for 36 Cancers in 185 Countries. CA Cancer J Clin. 2021; 71: 209-49.

2. Zhang B, Zhang Z, Huang Z, Chen $Y$, Chen M, Bie P, et al. 42,573 cases of hepatectomy in China: a multicenter retrospective investigation. Sci China Life Sci. 2018; 61: 660-70.

3. Popovic D, Vucic D, Dikic I. Ubiquitination in disease pathogenesis and treatment. Nat Med. 2014; 20: 1242-53.

4. Deng L, Meng T, Chen L, Wei W, Wang P. The role of ubiquitination in tumorigenesis and targeted drug discovery. Signal Transduct Target Ther. 2020; 5: 11.

5. Zhou X, Sun SC. Targeting ubiquitin signaling for cancer immunotherapy. Signal Transduct Target Ther. 2021; 6: 16.

6. Komander D, Clague MJ, Urbe S. Breaking the chains: structure and function of the deubiquitinases. Nat Rev Mol Cell Biol. 2009; 10: 550-63.

7. Chen $\mathrm{YJ}, \mathrm{Wu} \mathrm{H}$, Shen $\mathrm{XZ}$. The ubiquitin-proteasome system and its potential application in hepatocellular carcinoma therapy. Cancer Lett. 2016; 379: 245-52.

8. Tu K, Yang W, Li C, Zheng X, Lu Z, Guo C, et al. Fbxw7 is an independent prognostic marker and induces apoptosis and growth arrest by regulating YAP abundance in hepatocellular carcinoma. Mol Cancer. 2014; 13: 110.

9. $\mathrm{Xu} \mathrm{Q}, \mathrm{Tu} \mathrm{J}$, Dou C, Zhang J, Yang L, Liu X, et al. HSP90 promotes cell glycolysis, proliferation and inhibits apoptosis by regulating PKM2 abundance via Thr-328 phosphorylation in hepatocellular carcinoma. Mol Cancer. 2017; 16: 178

10. He $\mathrm{H}, \mathrm{Wu} \mathrm{Z}, \mathrm{Li} \mathrm{S}$, Chen $\mathrm{K}$, Wang $\mathrm{D}$, Zou $\mathrm{H}$, et al. TRAF7 enhances ubiquitin-degradation of KLF4 to promote hepatocellular carcinoma progression. Cancer Lett. 2020; 469: 380-9.

11. Ma Y, Li K, Li S, Liang B, Liu Q, Mo Z. Prognostic value of ubiquitin-conjugating enzyme E2 S overexpression in hepatocellular carcinoma. Int J Biol Macromol. 2018; 119: 225-31.

12. Zhang RY, Liu ZK, Wei D, Yong YL, Lin $\mathrm{P}$, Li H, et al. UBE2S interacting with TRIM28 in the nucleus accelerates cell cycle by ubiquitination of p27 to promote hepatocellular carcinoma development. Signal Transduct Target Ther. 2021; 6: 64 .

13. Tao NN, Zhang ZZ, Ren JH, Zhang J, Zhou YJ, Wai Wong VK, et al Overexpression of ubiquitin-conjugating enzyme E2 L3 in hepatocellular carcinoma potentiates apoptosis evasion by inhibiting the GSK3beta/p65 pathway. Cancer Lett. 2020; 481: 1-14.

14. Liang K, Volk AG, Haug JS, Marshall SA, Woodfin AR, Bartom ET, et al. Therapeutic Targeting of MLL Degradation Pathways in MLL-Rearranged Leukemia. Cell. 2017; 168: 59-72 e13.

15. Mashtalir N, Daou S, Barbour H, Sen NN, Gagnon J, Hammond-Martel I, et al. Autodeubiquitination protects the tumor suppressor BAP1 from cytoplasmic sequestration mediated by the atypical ubiquitin ligase UBE2O. Mol Cell. 2014; 54: 392-406.

16. Vila IK, Yao Y, Kim G, Xia W, Kim H, Kim SJ, et al. A UBE2O-AMPKalpha2 Axis that Promotes Tumor Initiation and Progression Offers Opportunities for Therapy. Cancer Cell. 2017; 31: 208-24.

17. Xu Y, Zhang Z, Li J, Tong J, Cao B, Taylor $\mathrm{P}$, et al. The ubiquitin-conjugating enzyme UBE2O modulates c-Maf stability and induces myeloma cell apoptosis. J Hematol Oncol. 2017; 10: 132.

18. Liu $\mathrm{X}, \mathrm{Ma} F$, Liu $\mathrm{C}$, Zhu $\mathrm{K}, \mathrm{Li} \mathrm{W}, \mathrm{Xu} \mathrm{Y}$, et al. UBE2O promotes the proliferation, EMT and stemness properties of breast cancer cells through the UBE2O/AMPKalpha2/mTORC1-MYC positive feedback loop. Cell Death Dis. 2020; 11: 10.

19. Huang $Y$, Yang $X$, Lu Y, Zhao $Y$, Meng R, Zhang S, et al. UBE2O targets Mxi1 for ubiquitination and degradation to promote lung cancer progression and radioresistance. Cell Death Differ. 2021; 28: 671-84.

20. Vila IK, Park MK, Setijono SR, Yao $Y$, Kim $H$, Badin PM, et al A muscle-specific UBE2O/AMPKalpha2 axis promotes insulin resistance and metabolic syndrome in obesity. JCI Insight. 2019; 4: e128269.

21. Dou C, Sun L, Wang L, Cheng J, Wu W, Zhang $C$, et al. Bromodomain-containing protein 9 promotes the growth and metastasis of human hepatocellular carcinoma by activating the TUFT1/AKT pathway. Cell Death Dis. 2020; 11: 730.

22. Gao S, Chen T, Li L, Liu X, Liu Y, Zhao J, et al. Hypoxia-Inducible Ubiquitin Specific Peptidase 13 Contributes to Tumor Growth and Metastasis via Enhancing the Toll-Like Receptor 4/Myeloid Differentiation Primary Response Gene 88/Nuclear Factor-kappaB Pathway in Hepatocellular Carcinoma. Front Cell Dev Biol. 2020; 8: 587389. 
23. Wang $\mathrm{Y}$, Yang $\mathrm{L}$, Chen $\mathrm{T}$, Liu $\mathrm{X}$, Guo $\mathrm{Y}$, Zhu $\mathrm{Q}$, et al. A novel IncRNA MCM3AP-AS1 promotes the growth of hepatocellular carcinoma by targeting miR-194-5p/FOXA1 axis. Mol Cancer. 2019; 18: 28.

24. Tang Z, Li C, Kang B, Gao G, Zhang Z. GEPIA: a web server for cancer and normal gene expression profiling and interactive analyses. Nucleic Acids Res. 2017; 45: W98-W102.

25. Chandrashekar DS, Bashel B, Balasubramanya SAH, Creighton CJ, Ponce-Rodriguez I, Chakravarthi B, et al. UALCAN: A Portal for Facilitating Tumor Subgroup Gene Expression and Survival Analyses. Neoplasia. 2017; 19: 649-58.

26. Lee CW, Wong LL, Tse EY, Liu HF, Leong VY, Lee JM, et al. AMPK promotes p53 acetylation via phosphorylation and inactivation of SIRT1 in liver cancer cells. Cancer Res. 2012; 72: 4394-404.

27. Wang YS, Du L, Liang X, Meng P, Bi L, Wang YL, et al. Sirtuin 4 Depletion Promotes Hepatocellular Carcinoma Tumorigenesis Through Regulating Adenosine-Monophosphate-Activated Protein Kinase Alpha/Mammalian Target of Rapamycin Axis in Mice. Hepatology. 2019; 69: 1614-31.

28. Meng H, Li G, Wei W, Bai Y, Feng Y, Fu M, et al. Epigenome-wide DNA methylation signature of benzo[a]pyrene exposure and their mediation roles in benzo[a]pyrene-associated lung cancer development. J Hazard Mater. 2021; 416: 125839 .

29. Zhang X, Zhang J, Bauer A, Zhang L, Selinger DW, Lu CX, et al. Fine-tuning BMP7 signalling in adipogenesis by UBE2O/E2-230K-mediated monoubiquitination of SMAD6. EMBO J. 2013; 32: 996-1007.

30. Shen JH, Chen PH, Liu HD, Huang DA, Li MM, Guo K. HSF1/AMPKalpha2 mediated alteration of metabolic phenotypes confers increased oxaliplatin resistance in HCC cells. Am J Cancer Res. 2019; 9: 2349-63.

31. Fang G, Zhang P, Liu J, Zhang X, Zhu X, Li R, et al. Inhibition of GSK-3beta activity suppresses HCC malignant phenotype by inhibiting glycolysis via activating AMPK/mTOR signaling. Cancer Lett. 2019; 463: 11-26.

32. Gao L, Lv G, Li R, Liu WT, Zong C, Ye F, et al. Glycochenodeoxycholate promotes hepatocellular carcinoma invasion and migration by AMPK/mTOR dependent autophagy activation. Cancer Lett. 2019; 454: 215-23.

33. Zhang ZY, Hong D, Nam SH, Kim JM, Paik YH, Joh JW, et al. SIRT1 regulates oncogenesis via a mutant p53-dependent pathway in hepatocellular carcinoma. J Hepatol. 2015; 62: 121-30.

34. Saxton RA, Sabatini DM. mTOR Signaling in Growth, Metabolism, and Disease. Cell. 2017; 168: 960-76.

35. Fantus D, Rogers NM, Grahammer F, Huber TB, Thomson AW. Roles of mTOR complexes in the kidney: implications for renal disease and transplantation. Nat Rev Nephrol. 2016; 12: 587-609.

36. Wang C, Cigliano A, Jiang L, Li X, Fan B, Pilo MG, et al. 4EBP1/eIF4E and p70S6K/RPS6 axes play critical and distinct roles in hepatocarcinogenesis driven by AKT and N-Ras proto-oncogenes in mice. Hepatology. 2015; 61: 200-13.

37. Yu XN, Chen H, Liu TT, Wu J, Zhu JM, Shen XZ. Targeting the mTOR regulatory network in hepatocellular carcinoma: Are we making headway? Biochim Biophys Acta Rev Cancer. 2019; 1871: 379-91.

38. Zhao X, Jiang P, Deng X, Li Z, Tian F, Guo F, et al. Inhibition of mTORC1 signaling sensitizes hepatocellular carcinoma cells to glycolytic stress. Am J Cancer Res. 2016; 6: 2289-98.

39. Sun L, Liu S, Wang X, Zheng X, Chen Y, Shen H. eIF6 promotes the malignant progression of human hepatocellular carcinoma via the mTOR signaling pathway. J Transl Med. 2021; 19: 216.

40. Adebayo Michael AO, Ko S, Tao J, Moghe A, Yang H, Xu M, et al. Inhibiting Glutamine-Dependent mTORC1 Activation Ameliorates Liver Cancers Driven by beta-Catenin Mutations. Cell Metab. 2019; 29: 1135-50 e6.

41. Tao J, Zhang $\mathrm{R}$, Singh $\mathrm{S}$, Poddar $\mathrm{M}, \mathrm{Xu} \mathrm{E}$, Oertel $\mathrm{M}$, et al. Targeting beta-catenin in hepatocellular cancers induced by coexpression of mutant beta-catenin and K-Ras in mice. Hepatology. 2017; 65: 1581-99.

42. Tao J, Krutsenko Y, Moghe A, Singh S, Poddar M, Bell A, et al. Nuclear factor erythroid 2-related factor 2 and beta-Catenin Coactivation in Hepatocellular Cancer: Biological and Therapeutic Implications. Hepatology. 2021; 74: 741-59. 